\title{
RetroXpert: Decompose Retrosynthesis Prediction like A Chemist
}

\author{
Chaochao Yan ${ }^{* \dagger}$ \\ University of Texas at Arlington \\ chaochao yan@mavs. uta.edu
}

\author{
Qianggang Ding *t \\ Tsinghua University
}

Shuangjia Zheng ${ }^{\dagger}$

Sun Yat-sen University

\author{
Jinyu Yang ${ }^{\dagger}$ \\ University of Texas at Arlington
}

\author{
Peilin Zhao \\ Tencent AI Lab
}

Junzhou Huang

University of Texas at Arlington

Tencent AI Lab

\author{
Yang Yu \\ Tencent AI Lab
}

\begin{abstract}
Retrosynthesis is the process of recursively decomposing target molecules into available building blocks. It plays an important role in solving problems in organic synthesis planning. To automate the retrosynthesis analysis, many retrosynthesis prediction methods have been proposed. However, most of them are cumbersome and lack interpretability about their predictions. In this paper, we devise a novel template-free algorithm, RetroXpert, for automatic retrosynthetic expansion by automating the procedure that chemists used to do. Our method disassembles retrosynthesis into two steps: i) we identify the potential reaction center within the target molecule through a graph neural network and generate intermediate synthons; and ii) we predict the associated reactants based on the obtained synthons via a reactant generation model. While outperforming the state-of-the-art baselines by a significant margin, our model also provides chemically reasonable interpretation.
\end{abstract}

\section{Introduction}

Retrosynthesis of the desired compound is commonly constructed by recursively decomposing it into a set of available reaction building blocks. This analysis mode was formalized in the pioneering work [1,2] and now becomes one of the fundamental paradigms in the modern chemical society. Retrosynthesis is challenging, in part due to the huge size of the search space and incomplete understanding of the reaction mechanism. Besides, retrosynthesis is typically undertaken by human experts, and therefore it is a subjective process and requires considerable expertise and experience. Last but not least, a molecule may have lots of possible retrosynthetic routes, and it is challenging to select the most appropriate one since the feasibility of a route is often determined by the global chemical structure of the molecule.

In this work, we focus on the "single-step " version (predict possible reactants given the product) of retrosynthesis following previous methods $[3,4,5]$. Our method can be decomposed into two subtasks [1, 6]: i) Breaking down the given target molecule into a set of synthons which are hypothetical

\footnotetext{
${ }^{*}$ Both authors contribute equally to the work.

${ }^{\dagger}$ This work is done when Chaochao Yan, Qianggang Ding, Shuangjia Zheng, and Jinyu Yang work as interns at Tencent AI Lab.
}

Preprint. Under review. 
units representing potential starting reactants in the retrosynthesis of the target; and ii) Calibrating the obtained synthons into a set of reactants, each of which corresponds to an available molecule.

Previously, many computational methods [7, 8, 9, 3, 10, 4] have been developed to assist in designing synthetic routes for novel molecules, among which most are dependent on hand-coded reaction templates. Template-based methods $[9,10,4]$ propose to automatically extract reaction templates from the reaction databases and choose appropriate templates to apply to the target. The key process of these approaches is to select relevant templates for the target molecule. However, these methods can only infer reactions within the chemical space covered by the template database, preventing them from discovering novel reactions [11]. Moreover, it is infeasible to manually encode all the synthesis routes in practice considering the exponential growth in the number of reactions. Besides, these methods heavily rely on the predefined atom dictionary to map atoms between reactants and products, which is still a nontrivial open problem [12,13]. Commonly used tools for identifying atom-mapping are based on expert rules and templates, which seems to get stuck in an infinite loop [14].

On the other hand, template-free methods [3,5] simply treat the retrosynthesis as a machine translation problem, since molecules can be represented as SMILES [15]. Although simple and expressive, these models do not fit into the chemists' analytical process and lack interpretability behind their predictions. Besides, such approaches fail to consider rich chemistry knowledge within the chemical reactions. For example, the generation order of reactants is undetermined in $[3,5]$ since they ignore the correlation between synthons and reactants, resulting in slower and inferior model convergence.

To overcome these challenges, inspired by the expert experience from chemists, we devise a two-step framework named as RetroXpert (Retrosynthesis eXpert) to automate the procedure that chemists used to do for retrosynthesis prediction. Our model tackles it in two steps as shown in Figure 1. Firstly, we propose to identify the potential reaction center within the target molecule using a novel Edge-enhanced Graph Attention Network (EGAT). The reaction center is referred to as the set of bonds that will be disconnected in the retrosynthesis. Synthons can be obtained by splitting the target molecule according to the reaction center. Secondly, the Reactant Generation Network (RGN) predicts associated reactants given the target molecule and synthons. Different from previous methods [3,5], the reactant generation order can be uniquely decided in our method, thanks to the intermediate synthons. What is more, we find the robustness of the RGN plays an important role. To robustify the RGN, we propose to augment the training data of RGN by incorporating unsuccessful predicted synthons. Our main contributions can be summarized as follows:

1) We propose to identify the potential reaction center with a novel Edge-enhanced Graph Attention Network (EGAT) which is largely dependent on chemical knowledge.

2) By splitting the target molecule into synthons, our RGN is able to determine the generation order of reactants. We further propose to augment training data by introducing unsuccessfully predicted synthons, which makes RGN robust and achieves significant improvement.

3) On the standard USPTO-50K dataset [16], our method achieves 70.4\% and 65.5\% Top-1 accuracy when $\mathrm{w} /$ and wo/ reaction type, respectively, which outperforms SOTA accuracy $63.2 \%(\mathrm{w} /)$ and $52.6 \%(\mathrm{wo} /)$ reported in [4] by a large margin.

\section{Methodology}

Given a molecule graph $\mathbf{G}$ with $N$ nodes (atoms), we denotes the matrix representation of node features as $X \in \mathbb{R}^{N \times M}$, the tensor representation of edge features as $E \in \mathbb{R}^{N \times N \times L}$, and the adjacency matrix as $A \in\{0,1\}^{N \times N} . M$ and $L$ are the feature dimensions of atoms and bonds, respectively. We denote as $P, S, R$ the product, synthons, and reactants in the reaction formulation, respectively. The "single-step " retrosynthesis problem can be described as given the desired product $P$, seeking for a set of reactants $R=\left\{R_{1}, R_{2}, \ldots, R_{n}\right\}$ that can produce the major product $P$ through a valid chemical reaction. It is denoted as $P \rightarrow R$ (predict $R$ given $P$ ), which is the reverse process of the forward reaction prediction problem that predicts the outcome products given a set of reactants.

As illustrated in Figure 1, our method decomposes the retrosynthesis task $(P \rightarrow R)$ into two closely dependent steps reaction center identification $(P \rightarrow S)$ and reactant generation $(S \rightarrow R)$. The first step is to identify the potential reaction bonds which will be disconnected during the reaction, then we can break down the product $P$ into a set of intermediate synthons $S=\left\{S_{1}, S_{2}, \ldots, S_{n}\right\}$. Note that each synthon $S_{i}$ is actually the substructure of a reactant $R_{i}$. The second step is to transform synthons 

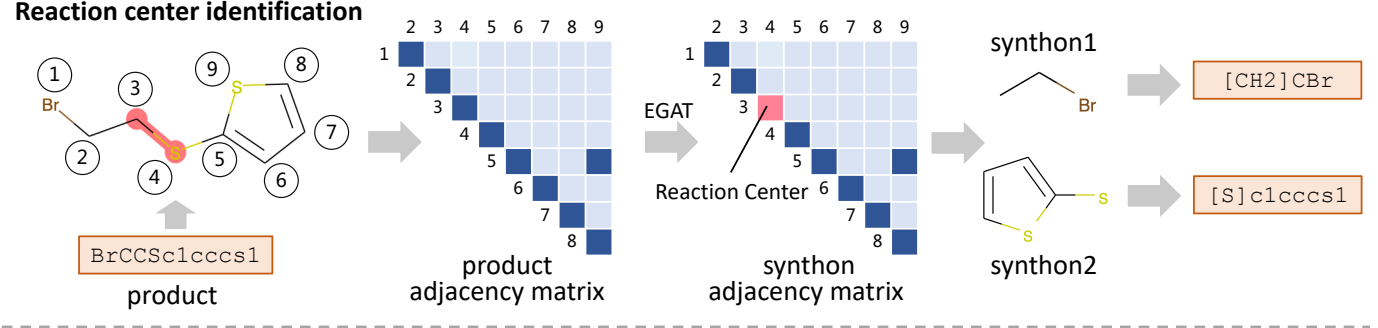

Reactants generation
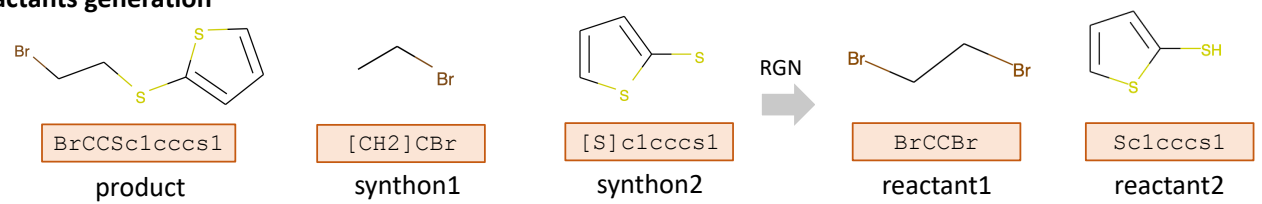

Figure 1: Pipeline overview. We conduct retrosynthesis in two dependent steps reaction center identification and reactant generation. The first step aims to identify the reaction center and generates intermediate synthons. The second step is to generate the desired set of reactants. Note that a molecule can be represented in two equivalent representations: molecule graph and SMILES string.

$S=\left\{S_{1}, S_{2}, \ldots, S_{n}\right\}$ to associated reactants $R=\left\{R_{1}, R_{2}, \ldots, R_{n}\right\}$. Although the intermediate synthons are not needed, decomposing the original retrosynthesis task $(P \rightarrow R)$ into two dependent procedures can have multiple benefits, which will be elaborated thoroughly in the following sections.

\subsection{EGAT for reaction center identification}

We treat the reaction center identification as a graph-to-graph transformation problem which is similar to the forward reaction outcome prediction [17]. To achieve this, we propose a graph neural network named Edge-enhanced Graph Attention Network (EGAT) which takes the molecule graph $\mathbf{G}$ as input and predicts disconnection probability for each bond, and this is the main task. Since a product may be produced by different reactions, there can be multiple reaction centers for a given product and each reaction center corresponds to a different reaction. Current message passing neural networks [18] are shallow and capture only local structure information for each node, and it is difficult to distinguish multiple reaction centers without global information. To alleviate the problem, we add a graph-level auxiliary task to predict the total number of disconnection bonds.

As shown in Figure 2, distinct from the Graph Attention Network (GAT) [19] which is designed to learn node and graph-level embeddings, our proposed EGAT also learns edge embedding. It identifies the reaction center by predicting the disconnection probability for each bond taking its edge embedding as input. Given the target $\mathbf{G}=\{A, E, X\}$, the EGAT layer computes node embedding $h_{i}^{\prime}$ and edge embedding $p_{i, j}^{\prime}$ from previous layer's embeddings $h_{i}$ and $p_{i, j}$ by following equations:

$$
\begin{aligned}
z_{i} & =\mathbf{W} h_{i} \\
c_{i, j} & =\operatorname{LeakyReLU}\left(\mathbf{a}^{T}\left[z_{i}\left\|z_{j}\right\| p_{i, j}\right]\right), \\
\alpha_{i, j} & =\frac{\exp \left(c_{i, j}\right)}{\sum_{k \in \mathcal{N}_{i}} \exp \left(c_{i, k}\right)} \\
h_{i}^{\prime} & =\sigma\left(\sum_{j \in \mathcal{N}_{i}} \alpha_{i, j} \mathbf{U}\left[z_{j} \| p_{i, j}\right]\right) \\
p_{i, j}^{\prime} & =\mathbf{V}\left[h_{i}^{\prime}\left\|h_{j}^{\prime}\right\| p_{i, j}\right]
\end{aligned}
$$

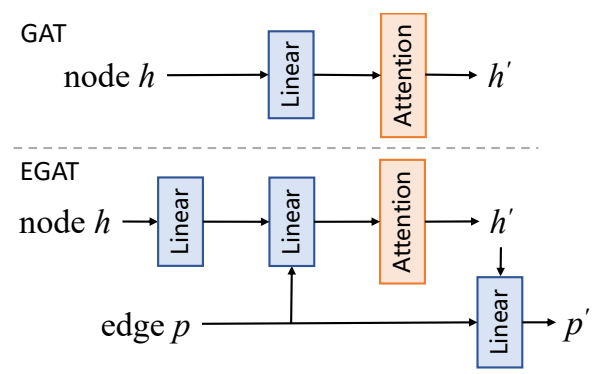

Figure 2: Embedding computation flows of GAT and EGAT. 
where $\mathbf{W} \in \mathbb{R}^{F^{\prime} \times F}, \mathbf{a} \in \mathbb{R}^{2 F^{\prime}+D}, \mathbf{U} \in \mathbb{R}^{F \times\left(F^{\prime}+D\right)}$, and $\mathbf{V} \in \mathbb{R}^{D \times(2 F+D)}$ are trainable parameters, $\|$ means concatenation operation, $\mathcal{N}_{i}$ is all neighbor nodes of the node $i, \alpha_{i, j}$ is the attention weight between the node $i$ and its neighbor node $j$, and $h_{i}^{\prime} \in \mathbb{R}^{F}$ as well as $p_{i, j}^{\prime} \in \mathbb{R}^{D}$ are the output node and edge representations, respectively. Initial input embeddings $h_{i}, p_{i, j}$ are the input node and edge feature vectors $x_{i}, e_{i, j}$, respectively, which will be detailed later, and in this special case the dimensions $F$ and $D$ equals to the dimensions of associated features, respectively.

After stacking multiple EGAT layers, we obtain the final edge representation $p_{i, j}$ for the chemical bond between nodes $i$ and $j$, as well as node representation $h_{i}$ for each node $i$. To predict the disconnection probability for a bond, we perform a fully-connected layer parameterized by $\mathbf{w}_{f c} \in \mathbb{R}^{D}$ and a Sigmoid activation layer to $p_{i, j}$ and its disconnection probability is $d_{i, j}=\operatorname{Sigmoid}\left(\mathbf{w}_{f c}^{T} \cdot p_{i, j}\right)$. Note that we can also apply the multi-head attention mechanism like the original GAT. Our optimization goal for bond disconnection prediction is to minimize the negative log-likelihood between prediction $d_{i, j}$ and ground-truth (GT) $y_{i, j} \in\{0,1\}$ through the binary cross entropy loss function:

$$
\mathcal{L}_{1}=-\frac{1}{K} \sum_{k=1}^{K} \sum_{a_{i, j} \in \mathbf{A}_{k}} a_{i, j}\left[\left(1-y_{i, j}\right) \log \left(1-d_{i, j}\right)+y_{i, j} \log \left(d_{i, j}\right)\right],
$$

where $K$ is the total number of reactions and bond $(i, j)$ exists if the associated adjacency element $a_{i, j}$ is nonzero. The GT $y_{i, j}=1$ means the bond $(i, j)$ is disconnected otherwise remaining the same. Bond disconnection labels can be obtained by comparing molecule graphs of target and reactants.

The graph-level representation $h_{G}=\operatorname{READOUT}\left(\left\{h_{i} \mid 1 \leq i \leq N\right\}\right)$, which is the output of the READOUT operation over all learned node representations, is the input of the auxiliary task. We adopts an arithmetic mean as the READOUT function $h_{G}=\frac{1}{N} \sum_{i=1}^{N} h_{i}$ and it works well in practice.

Similarly, a fully-connected layer parameterized by $\mathbf{W}_{s} \in \mathbb{R}^{\left(1+N_{\max }\right) \times F}$ and a Softmax activation function are applied to $h_{G}$ to predict the total number of disconnected bonds, which is solved as a classification problem here. Each category represents the exact number of disconnected bonds, so there are $1+N_{\max }$ classification categories. $N_{\max }$ is the maximum number of possible disconnected bonds in the retrosynthesis. We denote the Softmax output as $q=\operatorname{Softmax}\left(\mathbf{W}_{s} \cdot h_{G}\right)$. The total number of disconnected bonds for each target molecule is predicted as:

$$
n^{*}=\arg \max _{n}\left(q_{n}\right)=\arg \max _{n}\left(\operatorname{Softmax}\left(\mathbf{W}_{s} \cdot h_{G}\right)_{n}\right), 0 \leq n \leq N_{\text {max }} .
$$

The GT number of disconnected bonds for the molecule $k$ is denoted as $N_{k}$, the indicator function $\mathbb{1}\left(i, N_{k}\right)$ is 1 if $i$ equals to $N_{k}$ otherwise it is 0 , and the cross entropy loss for the auxiliary task:

$$
\mathcal{L}_{2}=\frac{1}{K} \sum_{k=1}^{K} \operatorname{Cross} \operatorname{Entropy}\left(N_{k}, q^{k}\right)=-\frac{1}{K} \sum_{k=1}^{K} \sum_{i=0}^{N_{\max }} \mathbb{1}\left(i, N_{k}\right) \log \left(q_{i}^{k}\right) .
$$

Finally, the overall loss function for the EGAT is $\mathcal{L}_{\mathrm{EGAT}}=\mathcal{L}_{1}+\alpha \mathcal{L}_{2}$, where $\alpha$ is fixed to 1 in our study since we empirically find that $\alpha$ is not a sensitive hype-parameter.

Atom and bond features. The atom feature consists of a series of general atom information such as atom type, hybridization, and formal charge, while the bond feature is composed of chemical bond information like bond type and conjugation (see Appendix B for details). These features are similar to those used in [20] which is for chemical property prediction. We compute these features using the open-source package RDKit [21]. To fully utilize the provided rich atom-mapping information of the USPTO datasets [16] [22], we add a semi-templates indicator to atom feature. Last not least, for retrosynthesis dataset with given reaction type, a class indicator is also added to the atom feature.

Semi-templates. For atom-mapped USPTO datasets, we extract reaction templates from reaction data like previous template-based methods [9, 10, 4]. However, we are not interested in full reaction templates since these templates are often too specific. There are as many as 11,647 templates for the USPTO-50K train data [4]. We keep only the product side templates instead, which we name as semi-templates. Since reaction templates are closely related to the exact reaction, we expect the semi-templates indicator can play a significant role in reaction center identification. 
The semi-template can be considered as a substructure pattern within a molecule. We build a database of semi-templates from training data and find all appeared semi-templates within each molecule. To build the indicator feature for an atom within the molecule, we mark the associated indicator bit for all atoms in the appeared semi-template. Note that each atom within a molecule may belong to several semi-templates since these semi-templates are not mutually exclusive. Although reaction templates are introduced, our method is still template-free since i) we only incorporate semi-templates and the EGAT does not rely on full templates to plan the retrosynthesis, and ii) our EGAT still works well in the absence of semi-templates, with slight performance degradation (Appendix D.2).

\subsection{Reactant generation network}

Once the reaction center has been identified, synthons can be obtained by applying bond disconnection to decompose the product graph. Since each synthon is basically a substructure within the reactant, we are informed of the total number of reactants and substructures of these reactants. The remaining task $S \rightarrow R$ is much simpler than the original $P \rightarrow R$ in which even number of reactants is unknown.

Specifically, task $S \rightarrow R$ is to generate the set of desired reactants given obtained synthons. Based on common knowledge of chemical reaction, we propose that the ideal RGN should meet the following three requirements: R1) be permutation invariant and generate the same set of reactants no matter the order of synthons, R2) all given information should be considered when generating any reactant, and R3) the generation of each reactant also depends on those previously generated reactants.

To fulfill these requirements, we represent molecules in SMILES and formulate $S \rightarrow R$ as a sequenceto-sequence prediction problem. We convert synthon graphs to SMILES representations using RDKit, though these synthons may be chemically invalid. As in Figure 3, source sequence is the concatenation of possible reaction types, canonical SMILES of the product, and associated synthons. The target sequence is the desired reactants arranged according to synthons.

We approximate the requirement $\mathrm{R} 1$ by augmenting train samples with reversely arranged synthons and reactants as shown in Figure 3. Our empirical studies demonstrate that such approximation works pretty well in practice. To satisfy the requirement R2, the encoder-decoder attention mechanism [23] [24] is employed, which allows each position in the target sequence attends to all positions in the source sequence. A similar masked selfattention mechanism [24], which masks future positions in the decoder, is adopted

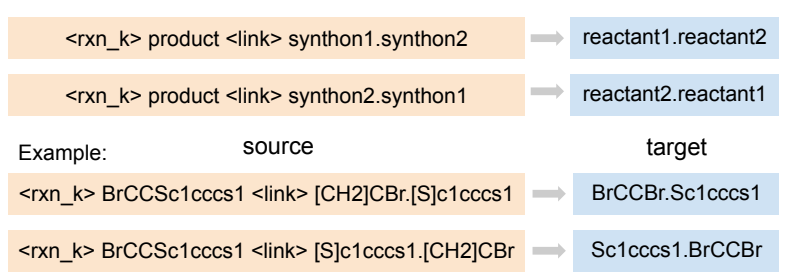

Figure 3: Illustration of source and target sequences. $<$ rxn_k $>$ is the $k$ th reaction type if applicable. The product and synthons are separated with a $<$ link $>$ token. SMILES strings are joined with a dot following RDkit. to make RGN meet the requirement R3.

Motivated by the success of Transformer [24] in natural machine translation, we build the RGN based on the Transformer module. Transformer is a sequence-to-sequence model equipped with two types of attention mechanisms: self-attention and encoder-decoder attention [24]. Transformer is also adapted for reaction outcome prediction [25] and retrosynthesis [5], in which both products and reactants are represented in SMILES. We include a brief description of Transformer in Appendix C.

Determine the generation order of reactants. For the first time, the generation order of reactants can be determined by aligning reactants in the target with synthons in the source, thanks to intermediate synthons which are associated with reactants uniquely. While the generation order of reactants is undetermined in previous methods [3] [5], which naively treats the sequence-to-sequence model as a black box. The uncertainty of the generation order makes their models hard to train.

Robustify the RGN. We find the EGAT suffers from distinguishing multiple coexisting reaction centers, which is the major bottleneck of our method. As a result of the failure of identifying the reaction center, the generated synthons are not the same as the GT. To make our RGN robust enough and able to predict the desired reactants even if the predicted synthons are different from the GT, we further augment RGN training data by including those unsuccessfully predicted synthons on training data. We do not reverse the order of synthons for these augmentation samples like in Figure 3. The 
intuition behind is that EGAT tends to make similar mistakes on training and test datasets since both datasets follow the same distribution. This method can make our RGN able to correct reaction center prediction error and generate the desired set of reactants.

\section{Experiments}

Dataset and preprocessing. We evaluate our method on USPTO-50K [16] and USPTO-full [22] to verify its effectiveness and scalability. USPTO-50K consists of 50K reactions annotated with 10 reaction types (see appendix A for type distribution), which is derived from USPTO granted patents [26]. It is widely used in previous retrosynthesis work. We adopt the same training/validation/test splits in 8:1:1 as [9,4]. For RGN training data, we add an extra $28 \mathrm{~K}$ samples of which synthons are reversed as shown in Figure 3 if there are at least two synthons. There are $68 \mathrm{~K}$ training samples for RGN, which is still denoted as USPTO-50K in the following content. The USPTO-full consists of 950K cleaned reactions from the USPTO 1976-2016 [22], which has 1,808,937 raw reactions without reaction types. We duplicate reactions with multiple products into multiple single-product ones. After removing invalid reactions (empty reactant and missing atom mappings) and deduplication, we can obtain $950 \mathrm{~K}$ reactions, which are randomly partitioned into training/validation/test sets in 8:1:1.

For the EGAT, we build molecule graphs using DGL [27] and extract atom and bond features with RDkit. The adjacency matrix of a molecule can be obtained using RDkit functional API. By comparing molecule graphs of product and reactants, we can identify disconnection bonds within the product graph and obtain training labels for both main and auxiliary tasks. This comparison can be easily done for atom-mapped reactions. For reactions without atom-mapping, a substructure matching algorithm in RDKit can be utilized to accomplish the comparison. We use RDChiral [28] to extract super general reaction templates, and obtain 1859 semi-templates for USPTO-50K training data. We filter semi-templates that appear less than twice and finally obtain 654 semi-templates. As for the RGN, we divide the product graph into synthon graphs according to the GT reaction center, then convert them into SMILES strings. The input sequence of RGN is the concatenation of the possible reaction type, product SMILES string, and synthon SMILES strings as illustrated in Figure 3.

Implementation. All reactions are represented in canonical SMILES, which are tokenized with the regular expression as in the previous paper [29]. We use DGL [27] and OpenNMT [30] to implement our EGAT and RGN models, respectively. As for the EGAT, we stack three identical four-head attentive layers of which the hidden dimension is 128. All embedding sizes in EGAT are set to 128, such as $F, F^{\prime}$, and $D$. The $N_{\max }$ is set to be two to cover $99.97 \%$ training samples. We train the EGAT on USPTO-50K for 80 epochs. EGAT parameters are optimized with Adam [31] with default settings, and the initial learning rate is 0.0005 and it is scheduled to multiply 0.2 every 20 epochs. We train the RNG for 300, 000 time steps, and it takes about 30 hours on two GTX 1080 Ti GPUs. We save a checkpoint of RGN parameters every 10,000 steps and averaging the last 10 checkpoints as the final model. We run all experiments for three times and report the means of their performance in default.

Evaluation metric. We use widely adopted Top- $N$ accuracy as the evaluation metric for retrosynthesis. Beam search [32] strategy is adapted to keep top K predictions throughout the reactant generation process. $\mathrm{K}$ is set to 50 in all experiments. The generated reactants are represented as canonical SMILES. A correct predicted set of reactants must be exactly the same as the GT reactants.

\subsection{Reaction center identification results}

To verify the effectiveness of edge-enhanced attention mechanism, we also include the ablation study by removing edge embedding $p_{i, j}$ when computing the coefficient $c_{i, j}=\operatorname{LeakyReLU}\left(\mathbf{a}^{T}\left[z_{i} \| z_{j}\right]\right)$. Results are reported in Table 1. The auxiliary task (Aux) can successfully predict the number of disconnection bonds for $99.2 \%$ test molecules given the reaction type (Type) while $86.4 \%$ if not given. As for the main task (Main) alone, its prediction accuracy is $74.4 \% \mathrm{w} /$ reaction type and $51.5 \%$ wo/ reaction type. However, if we adopt the prediction from the auxiliary task as the prior of the number of disconnection bonds, and select the most probable disconnection bonds (EGAT), then the prediction accuracy can be boosted to $86.0 \%(\mathrm{w} /)$ and $64.9 \%(\mathrm{wo} /)$, respectively. The edge-enhanced attention (EAtt) can consistently improve the model's performance in all tasks. The improvement is more significant when the reaction type is unknown, so our EGAT is more practical in real world 
applications without reaction types. This demonstrates that the reaction type information plays an important role in the retrosynthesis. The reactions of the same type usually share similar reaction patterns (involved atoms, bonds, and functional groups), it is much easier to recognize the reaction center if reaction type is given as the prior. We also verify the importance of semi-templates in Appendix D.2.

\subsection{Reactant prediction results}

To robustify the RGN as described in the paragraph Robustify the RGN, we also conduct the $P \rightarrow S$ prediction on the EGAT training data for USPTO-50K $(40 \mathrm{~K})$, and the prediction accuracy is $89.0 \%$ for the reaction type conditional setting. We can obtain about $4 \mathrm{~K}$ unsuccessful synthon predictions as augmentation samples (Aug), adding the original 68K RGN training data, the total RGN training data size is $72 \mathrm{~K}$. For the unconditional setting, the EGAT accuracy is $70.0 \%$ and there are $12 \mathrm{~K}$ augmentation samples, and the total RGN training size is $80 \mathrm{~K}$ in this case. We train RGN models on the USPTO-50K with/without the augmentation (Aug), and report results in Table 2.

RGN evaluation For the RGN evaluation, the RGN Table 1: Results of EGAT on USPTO-50K dataset. EAtt and Aux are the short for edgeenhanced attention and auxiliary task, respectively. EGAT consists of both main and auxiliary tasks. The prediction is binarized with a threshold of 0.5 if main task alone.

\begin{tabular}{ccccc}
\hline \multirow{2}{*}{ Type } & \multirow{2}{*}{ EAtt } & \multicolumn{3}{c}{ Accuracy $(\%)$} \\
\cline { 3 - 5 } & & Main & Aux & EGAT \\
\hline$\checkmark$ & $\boldsymbol{x}$ & 73.9 & 99.1 & 85.7 \\
$\checkmark$ & $\checkmark$ & $\mathbf{7 4 . 4}$ & $\mathbf{9 9 . 2}$ & $\mathbf{8 6 . 0}$ \\
\hline $\boldsymbol{x}$ & $\boldsymbol{x}$ & 50.0 & 86.1 & 64.3 \\
$\boldsymbol{x}$ & $\checkmark$ & $\mathbf{5 1 . 5}$ & $\mathbf{8 6 . 4}$ & $\mathbf{6 4 . 9}$ \\
\hline
\end{tabular}
input consists of the GT synthons. Therefore the results in Table 2 indicate the upper bound of our method's overall retrosynthesis performance. The proposed augmentation does not always improve the upper bound. Without given reaction type, the RGN generally performs worse with the augmentation due to the introduced dirty training samples. However, when given reaction type, this augmentation boosts its prediction accuracy. We presume that it is because the reaction type plays a significant role. The RGN learns to put more attention on the reaction type and product instead of synthons to generate the reactants.

Table 2: $S \rightarrow R$ prediction results. Aug denotes training data augmentation.

\begin{tabular}{ccccccccc}
\hline \multirow{2}{*}{ Type } & \multirow{2}{*}{ Aug } & \multirow{2}{*}{ Training size } & \multicolumn{6}{c}{ Top- $n$ accuracy $(\%)$} \\
\cline { 4 - 8 } & & & 1 & 3 & 5 & 10 & 20 & 50 \\
\hline $\boldsymbol{\checkmark}$ & $\boldsymbol{x}$ & $68 \mathrm{~K}$ & 72.9 & 86.5 & 88.3 & 89.5 & 90.4 & 91.6 \\
$\boldsymbol{}$ & $\boldsymbol{}$ & $72 \mathrm{~K}$ & $\mathbf{7 3 . 4}$ & $\mathbf{8 6 . 7}$ & $\mathbf{8 8 . 5}$ & $\mathbf{8 9 . 7}$ & $\mathbf{9 0 . 9}$ & $\mathbf{9 2 . 1}$ \\
\hline $\boldsymbol{x}$ & $\boldsymbol{x}$ & $68 \mathrm{~K}$ & $\mathbf{7 1 . 9}$ & $\mathbf{8 5 . 7}$ & $\mathbf{8 7 . 5}$ & $\mathbf{8 8 . 9}$ & $\mathbf{9 0 . 0}$ & $\mathbf{9 1 . 0}$ \\
$\boldsymbol{x}$ & $\boldsymbol{\checkmark}$ & $80 \mathrm{~K}$ & 70.9 & 84.6 & 86.4 & 88.2 & 89.4 & 90.6 \\
\hline
\end{tabular}

Retrosynthesis evaluation To evaluate the overall retrosynthesis prediction accuracy, the generated synthons from $P \rightarrow S$ instead of the GT are input into the RGN. In this way, we only need to compare the predicted reactants with the GT ones, without considering if the reaction center predictions correct or not. We report the retrosynthesis results in Tables 3. Our method RetroXpert achieves impressive performance on the test data. Specifically, when given reaction types, our proposed method achieves $70.4 \%$ Top- 1 accuracy, which outperforms the SOTA Top-1 accuracy $63.2 \%$ [4] by a large margin. Note that our Top-1 accuracy $70.4 \%$ is quite close to the upper bound $73.4 \%$ in Table 2, which indicates the proposed augmentation strategy in Robustify the RGN is considerably effective. As for results wo/ given reaction type, our model improves the SOTA Top-1 accuracy from 52.6\% [4] to $65.6 \%$. To verify the effectiveness of augmentation, we conduct ablation study in Appendix D.3.

While our method outperforms in Top- 1 , Top- 3 , and Top-5 accuracy, template-based methods GLN [4] and RetroSim [9] are better at Top-20 and Top-50 predictions since they enumerate multiple different reaction templates for each product to increase the hit rate. While our RetroXpert is currently designed to find the best set of reactants. To increase the diversity, we can design new strategies to enumerate multiple reaction centers for each product. This is left as the feature work. 
We also notice that the gap between Top- 2 and Top- 1 accuracy is around $10 \%$. After investigating these $10 \%$ predictions by experienced chemists from the synthetic chemistry perspective, we find about 9/10 these Top-1 predictions are actually reasonable (see Appendix E for more details). This indicates our method can learn general chemical reaction knowledge, which is beyond the given GT.

Table 3: Retrosynthesis results compared with the SOTA methods. NeuralSym [33] results are copied from [4]. *We run the self-implemented SCROP [5] and official implementation of GLN [4] on the USPTO-full.

\begin{tabular}{lcccccc}
\hline \multirow{2}{*}{ Methods } & \multicolumn{6}{c}{ Top- $n$ accuracy (\%) } \\
\cline { 2 - 7 } & 1 & 3 & 5 & 10 & 20 & 50 \\
\hline \multicolumn{6}{c}{ Reaction types given as prior on USPTO-50K } \\
\hline Seq2Seq [3] & 37.4 & 52.4 & 57.0 & 61.7 & 65.9 & 70.7 \\
RetroSim [9] & 52.9 & 73.8 & 81.2 & 88.1 & 91.8 & 92.9 \\
NeuralSym [33] & 55.3 & 76.0 & 81.4 & 85.1 & 86.5 & 86.9 \\
SCROP [5] & 59.0 & 74.8 & 78.1 & 81.1 & - & - \\
GLN [4] & 63.2 & 77.5 & 83.4 & $\mathbf{8 9 . 1}$ & $\mathbf{9 2 . 1}$ & $\mathbf{9 3 . 2}$ \\
RetroXpert & $\mathbf{7 0 . 4}$ & $\mathbf{8 3 . 4}$ & $\mathbf{8 5 . 3}$ & 86.8 & 88.1 & 89.3 \\
\hline \multicolumn{6}{c}{ Reaction type unknown on USPTO-50K } \\
\hline RetroSim [9] & 37.3 & 54.7 & 63.3 & 74.1 & 82.0 & 85.3 \\
NeuralSym [33] & 44.4 & 65.3 & 72.4 & 78.9 & 82.2 & 83.1 \\
SCROP [5] & 43.7 & 60.0 & 65.2 & 68.7 & - & - \\
GLN [4] & 52.6 & 68.0 & 75.1 & 83.1 & $\mathbf{8 8 . 5}$ & $\mathbf{9 2 . 1}$ \\
RetroXpert & $\mathbf{6 5 . 6}$ & $\mathbf{7 8 . 7}$ & $\mathbf{8 0 . 8}$ & $\mathbf{8 3 . 3}$ & 84.6 & 86.0 \\
\hline \multicolumn{6}{c}{ Retrosynthesis results on USPTO-full. } \\
\hline GLN* [4] & 39.0 & 50.1 & 55.3 & 61.3 & 65.9 & 69.1 \\
SCROP* [5] & 45.7 & 60.7 & 65.3 & 70.1 & 73.3 & 76.0 \\
RetroXpert & $\mathbf{4 9 . 4}$ & $\mathbf{6 3 . 6}$ & $\mathbf{6 7 . 6}$ & $\mathbf{7 1 . 6}$ & $\mathbf{7 4 . 6}$ & $\mathbf{7 7 . 0}$ \\
\hline
\end{tabular}

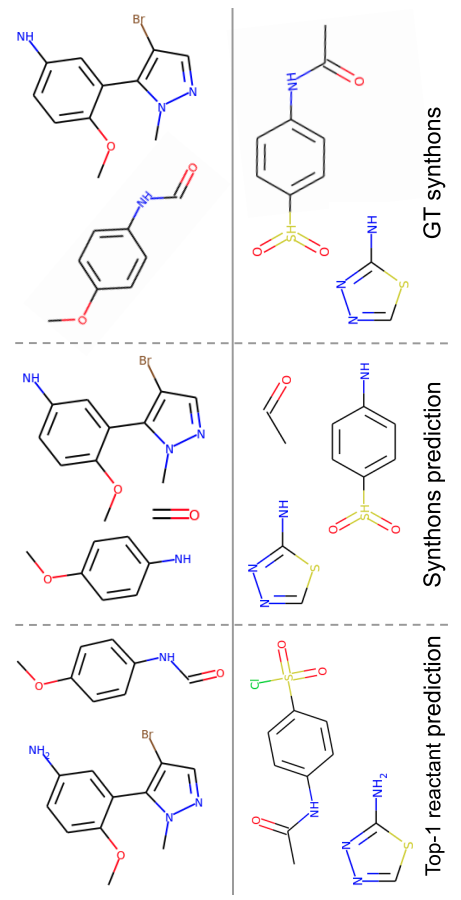

Figure 5: Robustness of the RGN. Top-1 prediction is the same to GT.

\section{Large scale experiments}

To demonstrate the scalability, we also experiment on the USPTO-full dataset, which consists of 760K training data. We extract 75,129 semi-templates and keep only 3,788 ones that appear at least 10 times. We set $N_{\max }$ as 5 to cover $99.87 \%$ training data. We obtain $1.35 \mathrm{M}$ training data after reversing synthons. The final accuracy of the $P \rightarrow S$ on training set is $60.5 \%$, and there are $0.3 \mathrm{M}$ unsuccessful synthon data and the total RNG training data size is $1.65 \mathrm{M}$. We train the RNG for 500,000 time steps on USPTO-full while keeping the other settings the same as those in section 3. We run the official implementation of GLN following their instructions [4], and the self-implemented SCROP [5] on the USPTO-full dataset. Experimental results are reported at the bottom of Table 3. Our method again significantly outperforms the SCROP and GLN, which demonstrates that our model scales well to the large real-world dataset. Note that template-free methods SCROP and RetroXpert outperform the GLN significantly, which may indicate the scalability of template-based methods is very limited.

\section{Prediction visualization}

For EGAT, how the auxiliary task helps to identify the reaction center is illustrated in Figure 5. Note that in the first example the two colored bonds and their surrounding structures are very similar. Current shallow GNNs consider only local information and fails to distinguish the true reaction center. Under the guidance of the auxiliary task, EGAT is able to identify the true reaction center. Figure 5 demonstrates the robustness of our method. Even if the predicted synthons are different from the GT, the RGN still successfully generates desired reactants. More illustrations in Appendix F. 


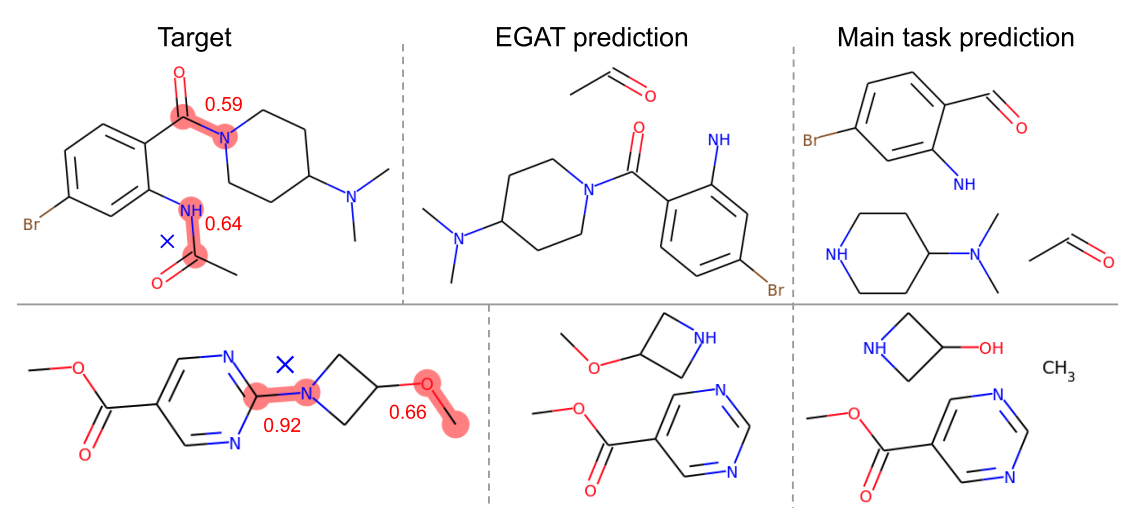

Figure 5: Importance of the auxiliary task. Pink indicates the reaction center along with disconnection probability in red predicted by the EGAT main task. Blue cross indicates the GT. Our EGAT successfully finds the desired reaction center under the guidance of the auxiliary task.

\section{Discussion}

One major common limitation of current retrosynthesis work is the lack of reasonable evaluation metrics. There may be multiple valid ways to synthesize a product, while the current evaluation metric considers only the given reaction. More evaluation metrics should be proposed in the future.

\section{References}

[1] EJ Corey and W Todd Wipke. Computer-assisted design of complex organic syntheses. Science, 166(3902):178-192, 1969.

[2] Elias James Corey. The logic of chemical synthesis: multistep synthesis of complex carbogenic molecules (nobel lecture). Angewandte Chemie International Edition in English, 30(5):455-465, 1991.

[3] Bowen Liu, Bharath Ramsundar, Prasad Kawthekar, Jade Shi, Joseph Gomes, Quang Luu Nguyen, Stephen Ho, Jack Sloane, Paul Wender, and Vijay Pande. Retrosynthetic reaction prediction using neural sequenceto-sequence models. ACS central science, 3(10):1103-1113, 2017.

[4] Hanjun Dai, Chengtao Li, Connor Coley, Bo Dai, and Le Song. Retrosynthesis prediction with conditional graph logic network. In Advances in Neural Information Processing Systems, pages 8870-8880, 2019.

[5] Shuangjia Zheng, Jiahua Rao, Zhongyue Zhang, Jun Xu, and Yuedong Yang. Predicting retrosynthetic reactions using self-corrected transformer neural networks. Journal of Chemical Information and Modeling, 2020.

[6] David A Pensak and EJ Corey. Lhasa—logic and heuristics applied to synthetic analysis. 1977.

[7] Clara D Christ, Matthias Zentgraf, and Jan M Kriegl. Mining electronic laboratory notebooks: analysis, retrosynthesis, and reaction based enumeration. Journal of chemical information and modeling, 52(7):17451756, 2012.

[8] Anders Bogevig, Hans-Jurgen Federsel, Fernando Huerta, Michael G Hutchings, Hans Kraut, Thomas Langer, Peter Low, Christoph Oppawsky, Tobias Rein, and Heinz Saller. Route design in the 21st century: The ic synth software tool as an idea generator for synthesis prediction. Organic Process Research \& Development, 19(2):357-368, 2015.

[9] Connor W Coley, Luke Rogers, William H Green, and Klavs F Jensen. Computer-assisted retrosynthesis based on molecular similarity. ACS central science, 3(12):1237-1245, 2017.

[10] Marwin HS Segler, Mike Preuss, and Mark P Waller. Planning chemical syntheses with deep neural networks and symbolic ai. Nature, 555(7698):604-610, 2018.

[11] Marwin HS Segler and Mark P Waller. Modelling chemical reasoning to predict and invent reactions. Chemistry-A European Journal, 23(25):6118-6128, 2017.

[12] William Lingran Chen, David Z Chen, and Keith T Taylor. Automatic reaction mapping and reaction center detection. Wiley Interdisciplinary Reviews: Computational Molecular Science, 3(6):560-593, 2013. 
[13] Wojciech Jaworski, Sara Szymkuć, Barbara Mikulak-Klucznik, Krzysztof Piecuch, Tomasz Klucznik, Michał Kaźmierowski, Jan Rydzewski, Anna Gambin, and Bartosz A Grzybowski. Automatic mapping of atoms across both simple and complex chemical reactions. Nature communications, 10(1):1-11, 2019.

[14] Nadine Schneider, Daniel M Lowe, Roger A Sayle, and Gregory A Landrum. Development of a novel fingerprint for chemical reactions and its application to large-scale reaction classification and similarity. Journal of chemical information and modeling, 55(1):39-53, 2015.

[15] David Weininger. Smiles, a chemical language and information system. 1. introduction to methodology and encoding rules. Journal of chemical information and computer sciences, 28(1):31-36, 1988.

[16] Nadine Schneider, Nikolaus Stiefl, and Gregory A Landrum. What's what: The (nearly) definitive guide to reaction role assignment. Journal of chemical information and modeling, 56(12):2336-2346, 2016.

[17] Wengong Jin, Connor Coley, Regina Barzilay, and Tommi Jaakkola. Predicting organic reaction outcomes with weisfeiler-lehman network. In Advances in Neural Information Processing Systems, pages 2607-2616, 2017.

[18] Justin Gilmer, Samuel S Schoenholz, Patrick F Riley, Oriol Vinyals, and George E Dahl. Neural message passing for quantum chemistry. In Proceedings of the 34th International Conference on Machine LearningVolume 70, pages 1263-1272. JMLR. org, 2017.

[19] Petar Veličković, Guillem Cucurull, Arantxa Casanova, Adriana Romero, Pietro Liò, and Yoshua Bengio. Graph Attention Networks. International Conference on Learning Representations, 2018.

[20] Kevin Yang, Kyle Swanson, Wengong Jin, Connor Coley, Philipp Eiden, Hua Gao, Angel Guzman-Perez, Timothy Hopper, Brian Kelley, Miriam Mathea, et al. Analyzing learned molecular representations for property prediction. Journal of chemical information and modeling, 59(8):3370-3388, 2019.

[21] Greg Landrum et al. Rdkit: Open-source cheminformatics. 2006.

[22] Daniel Lowe. Chemical reactions from us patents (1976-sep2016), 2018.

[23] Dzmitry Bahdanau, Kyunghyun Cho, and Yoshua Bengio. Neural machine translation by jointly learning to align and translate. arXiv preprint arXiv:1409.0473, 2014.

[24] Ashish Vaswani, Noam Shazeer, Niki Parmar, Jakob Uszkoreit, Llion Jones, Aidan N Gomez, Łukasz Kaiser, and Illia Polosukhin. Attention is all you need. In Advances in neural information processing systems, pages 5998-6008, 2017.

[25] Philippe Schwaller, Teodoro Laino, Théophile Gaudin, Peter Bolgar, Christopher A Hunter, Costas Bekas, and Alpha A Lee. Molecular transformer: A model for uncertainty-calibrated chemical reaction prediction. ACS central science, 5(9):1572-1583, 2019.

[26] Daniel Mark Lowe. Extraction of chemical structures and reactions from the literature. PhD thesis, University of Cambridge, 2012.

[27] Minjie Wang, Lingfan Yu, Da Zheng, Quan Gan, Yu Gai, Zihao Ye, Mufei Li, Jinjing Zhou, Qi Huang, Chao Ma, et al. Deep graph library: Towards efficient and scalable deep learning on graphs. arXiv preprint arXiv:1909.01315, 2019.

[28] Connor W Coley, William H Green, and Klavs F Jensen. Rdchiral: An rdkit wrapper for handling stereochemistry in retrosynthetic template extraction and application. Journal of chemical information and modeling, 59(6):2529-2537, 2019.

[29] Philippe Schwaller, Theophile Gaudin, David Lanyi, Costas Bekas, and Teodoro Laino. "found in translation": predicting outcomes of complex organic chemistry reactions using neural sequence-tosequence models. Chemical science, 9(28):6091-6098, 2018.

[30] Guillaume Klein, Yoon Kim, Yuntian Deng, Jean Senellart, and Alexander M. Rush. OpenNMT: Opensource toolkit for neural machine translation. In Proc. ACL, 2017.

[31] Diederik P Kingma and Jimmy Ba. Adam: A method for stochastic optimization. arXiv preprint arXiv:1412.6980, 2014.

[32] Christoph Tillmann and Hermann Ney. Word reordering and a dynamic programming beam search algorithm for statistical machine translation. Computational linguistics, 29(1):97-133, 2003.

[33] Marwin HS Segler and Mark P Waller. Neural-symbolic machine learning for retrosynthesis and reaction prediction. Chemistry-A European Journal, 23(25):5966-5971, 2017. 